\title{
A Novel Incorporate Algorithm of Concept Lattice
}

\author{
Xi Gong \\ School of Software Engineering, University of Science and Technology LiaoNing, Anshan, 114051, China \\ E-mail: askdjy05gx@163.com
}

\begin{abstract}
With the expansion of the research field, the research object of some original seemingly unrelated properties have been studied together. At this time, the number of attribute in formal context has changed. For the increased attributes, we need to construct a new concept lattice. The existing incremental building algorithms of concept lattice need the original formal context as the basis, with single attribute or a set of attribute of the object to rebuild the concept lattice. They can't effectively utilize these existing concept lattice that have not relation in attributes. Here, the paper presents one new algorithm for incorporating concept lattice based on the existed concept lattices. We can directly build the "together lattice" from bottom to top by direct product operation on the existed concept lattices and the mapping relation between the direct product lattice of two existed concept lattices and the "together lattice". Formal contexts that attribute sets have no intersection are fit for this algorithm.
\end{abstract}

Keywords-component: formal context; concept lattice; incorporating; direct product

\section{INTRODUCTION}

Since the formal concept analysis theory was put forward by German Wille[1] professor in 1982, with 30 years' development, the formal concept analysis and its core data structure concept lattice was proved to be an effective tool of knowledge discovery and data analysis .It has been widely used in the field of knowledge discovery, software engineering, information retrieval, information filtering etc[2-6]. How to improve the efficiency of constructing concept lattice is a hotspot problem. The existing algorithm to structure concept lattice can be divided into two categories: batch algorithm [7,8] and incremental algorithm [9-10]. Along with the information storage increase gradually, it often need a lot of formal context merge together to form larger formal context. The space and time complexity of concept lattice grows exponentially with the scale of the increase of the formal context. If we use the existing incremental algorithms to reconstruct the concept lattice of the new formal context, the method consumed a mass of memory and time. This algorithm directly utilizes two existing formal context corresponding to the original concept lattices to construct the total concept lattice that corresponding to the merged formal context and solves the problem of low utilization ratio of the original concept lattice.

\section{BASIC CONCEPTS AND BASIC THEOREMS[11]}

Definition 1 A formal context is composed of two sets $O$ and $A$ and a relation $I$ between $O$ and $A$. The elements of $O$ are called the objects and the elements of $A$ are called the attributes. In order to express that object $o$ is in relation $I$ with attribute $a$, we write $(o, a) \in I$ or oIa .

Definition 2 Given a set of objects $X \subseteq O$ and a set of attributes $Y \subseteq A$ from the formal context $(O, A, I)$, two operators can be defined as:

$$
f(X)=\{a \in A \mid \forall x \in X, x I a\} \quad g(Y)=\{o \in O \mid \forall y \in Y, o I y\}
$$

Definition 3 For $x \subseteq O, y \subseteq A$, a pair $(x, y)$ such that $f(x)=y, g(y)=x$, is called a (formal) concept. For a concept $(x, y)$ the set $x$ is called the extent and the set $y$ the intent of the concept.

Definition 4 If $\left(x_{1}, y_{1}\right),\left(x_{2}, y_{2}\right)$ are two concepts of formal context $(O, A, I)$ with $x_{1} \subseteq x_{2}$, then $\left(x_{2}, y_{2}\right)$ is called the super concept of $\left(x_{1}, y_{1}\right)$, written as $\left(x_{1}, y_{1}\right) \leq\left(x_{2}, y_{2}\right)$.If $\left(x_{1}, y_{1}\right) \leq\left(x_{2}\right.$, $\left.y_{2}\right),\left(x_{1}, y_{1}\right) \neq\left(x_{2}, y_{2}\right)$ then written as $\left(x_{1}, y_{1}\right)<\left(x_{2}, y_{2}\right)$.If there is no $\left(x_{3}, y_{3}\right)$ such that $\left(x_{1}, y_{1}\right)<\left(x_{3}, y_{3}\right)<\left(x_{2}, y_{2}\right)$ then $\left(x_{2}, y_{2}\right)$ is called as the father concept of $\left(x_{1}, y_{1}\right)$ and $\left(x_{1}, y_{1}\right)$ is called as the child concept of $\left(x_{2}, y_{2}\right)$. With respect to this partial order, the set of all formal concepts forms a complete lattice written as $L(O, A, I) . L(O, A, I)=(L$ $(O, A, I), \leq)$ is called the concept lattice of the formal context $(O, A, I)$.

Definition 5 The direct product of two partial order sets $\left(M_{1}, \leq\right)$ and $\left(M_{2}, \leq\right)$ is defined as a partial order set $\left(M_{1} \times M_{2}, \leq\right)$.the " $\leq$ " means that $\left(x_{1}, x_{2}\right) \leq\left(y_{1}, y_{2}\right): \Leftrightarrow x_{1} \leq y_{1}$ and $x_{2} \leq y_{2}$.

Theorem 1 concepts lattice $L(O, A, I)$ is a complete lattice. The infimum and supremum are defined as:

$$
\underset{t \in T}{\wedge}\left(A_{t}, B_{t}\right)=\left(\underset{t \in T}{\cap} A_{t}, f\left(\underset{t \in T}{\cap} A_{t}\right)\right) \underset{t \in T}{\vee}\left(A_{t}, B_{t}\right)=\left(g\left(\underset{t \in T}{\cap} B_{t}\right), \underset{t \in T}{\cap} B_{t}\right),
$$

$T$ is a index set.

\section{BASIC PRINCIPLE OF THE ALGORITHM}

In this paper, the original formal context is $\left(O, A_{1}, I_{1}\right)$. The concept lattice expressed by $L_{1}$. The additional attribute set in the formal context is $A_{2}$. Its corresponding formal context is $\left(O, A_{2}, I_{2}\right)$, the concept lattice expressed by $L_{2} \cdot L_{X}=L_{1} \times L_{2}$ is the direct product of $L_{1}$ and $L_{2} . L_{X}$ is a complete lattice. $\left(c_{1}, c_{2}\right)$ is the element of $L_{X} \cdot c_{1}$ is the concept of $I_{1}$ as well as $c_{2}$ is the concept of $I_{2}$. The set expressed by $C_{X}$. The total concept lattice is expressed by $L$. $C$ expresses the set of all elements of $L$.When $A_{1} \cap A_{2}=\varnothing$, this algorithm is applicative. 
Definition $6 \varphi: \quad C \rightarrow C_{X}$ is a mapping that associates a concept of the total lattice $L$ to a concept of the direct product lattice $L_{X}$.

$$
\varphi((x, y))=\left(\left(g\left(y \cap A_{1}\right), y \cap A_{1}\right),\left(g\left(y \cap A_{2}\right), y \cap A_{2}\right)\right)
$$

Proposition 1 The mapping $\varphi$ is an order embedded that associates $L$ to $L_{X}$ and injective.

Proof: Consider $\left(x_{1}, y_{1}\right),\left(x_{2}, y_{2}\right) \in C$ with $\left(x_{1}, y_{1}\right) \leq\left(x_{2}, y_{2}\right)$ then $\varphi\left(\left(x_{1}, y_{1}\right)\right)=\left(\left(g\left(y_{1} \cap A_{1}\right), y_{1} \cap A_{1}\right),\left(g\left(y_{1} \cap A_{2}\right), y_{1} \cap A_{2}\right)\right)$ $\varphi\left(\left(x_{2}, y_{2}\right)\right)=\left(\left(g\left(y_{2} \cap A_{1}\right), y_{2} \cap A_{1}\right),\left(g\left(y_{2} \cap A_{2}\right), y_{2} \cap A_{2}\right)\right)$

For $\left(x_{1}, y_{1}\right) \leq\left(x_{2}, y_{2}\right) \Rightarrow y_{2} \subseteq y_{1} \Rightarrow y_{2} \cap A_{1} \subseteq y_{1} \cap A_{1}$, $y_{2} \cap A_{2} \subseteq y_{1} \cap A_{2} \Rightarrow\left(g\left(y_{1} \cap A_{1}\right), y_{1} \cap A_{1}\right) \leq\left(g\left(y_{2} \cap A_{1}\right), y_{2} \cap A_{1}\right)$ and $\left(g\left(y_{1} \cap A_{2}\right), y_{1} \cap A_{2}\right) \leq\left(g\left(y_{2} \cap A_{2}\right), y_{2} \cap A_{2}\right)$ then $\varphi\left(\left(x_{1}, y_{1}\right)\right)$ $\leq \varphi\left(\left(x_{2}, y_{2}\right)\right)$. Obviously, the mapping $\varphi$ is order-preserving .

Consider $\varphi\left(\left(x_{1}, y_{1}\right)\right) \leq \varphi\left(\left(x_{2}, y_{2}\right)\right)$ with $\left(x_{1}, y_{1}\right),\left(x_{2}, y_{2}\right) \in C$ then $\left(g\left(y_{1} \cap A_{1}\right), y_{1} \cap A_{1}\right) \leq\left(g\left(y_{2} \cap A_{1}\right), y_{2} \cap A_{1}\right) \Rightarrow y_{2} \cap A_{1} \subseteq y_{1} \cap A_{1}$ and $\left(g\left(y_{1} \cap A_{2}\right), y_{1} \cap A_{2}\right) \leq\left(g\left(y_{2} \cap A_{2}\right), y_{2} \cap A_{2}\right) \Rightarrow y_{2} \cap A_{2} \subseteq y_{1} \cap A_{2}$ For $\left(y_{2} \cap A_{1}\right) \cup\left(y_{2} \cap A_{2}\right) \subseteq\left(y_{1} \cap A_{1}\right) \cup\left(y_{1} \cap A_{2}\right) \Rightarrow y_{2} \cap\left(A_{1} \cup A_{2}\right) \subseteq y_{1} \cap\left(A_{1}\right.$ $\left.\cup A_{2}\right) \Rightarrow y_{2} \cap A \subseteq y_{1} \cap A$. For $y_{1} \subseteq A$ and $y_{2} \subseteq A \Rightarrow y_{2} \subseteq y_{1} \Rightarrow\left(x_{1}, y_{1}\right) \leq\left(x_{2}\right.$, $\left.y_{2}\right)$. So, the mapping $\varphi$ is an order embedded .

Next, we proof the mapping $\varphi$ is injective.

Consider $\varphi\left(\left(x_{1}, y_{1}\right)\right)=\varphi\left(\left(x_{2}, y_{2}\right)\right)$ with $\left(x_{1}, y_{1}\right),\left(x_{2}, y_{2}\right) \in C$ then $\left(g\left(y_{1} \cap A_{1}\right), y_{1} \cap A_{1}\right)=\left(g\left(y_{2} \cap A_{1}\right), y_{2} \cap A_{1}\right) \Rightarrow y_{1} \cap A_{1}=y_{2} \cap A_{1}$ $\left(g\left(y_{1} \cap A_{2}\right), y_{1} \cap A_{2}\right)=\left(g\left(y_{2} \cap A_{2}\right), y_{2} \cap A_{2}\right) \Rightarrow y_{1} \cap A_{2}=y_{2} \cap A_{2}$

For $\left(y_{1} \cap A_{1}\right) \cup\left(y_{1} \cap A_{2}\right)=\left(y_{2} \cap A_{1}\right) \cup\left(y_{2} \cap A_{2}\right) \Rightarrow y_{1} \cap\left(A_{1} \cup A_{2}\right)=y_{2} \cap$ $\left(A_{1} \cup A_{2}\right) \Rightarrow y_{2} \cap A=y_{1} \cap A \quad$.For $\quad y_{1} \subseteq A \quad$ and $\quad y_{2} \subseteq A \Rightarrow y_{1}=y_{2} \Rightarrow$ $\left(x_{1}, y_{1}\right)=\left(x_{2}, y_{2}\right)$. So, the mapping $\varphi$ is injective.

Proposition 2 The mapping $\varphi$ is supremum-preserving.

Proof: Let $X=\left\{\left(x_{t}, y_{t}\right) \mid T\right.$ is an index set, $\forall t \in T,\left(x_{t}, y_{t}\right)$ is a concept of total lattice $L\} \subseteq C$, so $X$ is a subset of $C$, then $\vee X=\left(g\left(\underset{t \in T}{\cap} y_{t}\right), \underset{t \in T}{\cap} y_{t}\right), \varphi(\vee X)=\varphi\left(g\left(\underset{t \in T}{\cap} y_{t}\right), \underset{t \in T}{\cap} y_{t}\right)=((g$ $\left.\left.\left(\cap \in y_{t} \cap A_{1}\right), \underset{t \in T}{\cap} y_{t} \cap A_{1}\right),\left(g\left(\underset{t \in T}{\cap} y_{t} \cap A_{2}\right), \cap y_{t} \cap A_{2}\right)\right) \cdot \varphi(X)=\left\{\left(g\left(y_{t} \cap\right.\right.\right.$ $\left.\left.A_{1}\right), y_{t} \cap A_{1}\right),\left(g\left(y_{t} \cap A_{2}\right), y_{t} \cap A_{2}\right) \mid T$ is an index set, $\forall t \in T,\left(x_{t}\right.$, $\left.y_{t}\right)$ is a concept of total lattice $L$.

$\vee \varphi(X)=\left(\left(g\left(\underset{t \in T}{\cap}\left(y_{t} \cap A_{1}\right)\right), \underset{t \in T}{\cap}\left(y_{t} \cap A_{1}\right)\right),\left(g\left(\underset{t \in T}{\cap}\left(y_{t} \cap A_{2}\right)\right), \cap\left(y_{t} \cap A_{2}\right)\right)\right)$ $=\left(\left(g\left(\underset{t \in T}{\cap} y_{t} \cap A_{1}\right), \underset{t \in T}{\cap} y_{t} \cap A_{1}\right),\left(g\left(\underset{t \in T}{\cap} y_{t} \cap A_{2}\right), \underset{t \in T}{\cap} y_{t} \cap A_{2}\right)\right)=\varphi(\vee X)$. So mapping $\varphi$ is supremum-preserving.

Definition $7 \psi: C_{X} \rightarrow C$ is a mapping that associates a concept of the direct product lattice $L_{X}$ to a concept of the total lattice $L$.

$$
\psi\left(\left(x_{1}, y_{1}\right),\left(x_{2}, y_{2}\right)\right)=\left(x_{1} \cap x_{2}, f\left(x_{1} \cap x_{2}\right)\right)
$$

Proposition 3 The mapping $\psi$ is order-preserving and infimum-preserving.

Proof: If $\left(\left(x_{1}, y_{1}\right),\left(x_{2}, y_{2}\right)\right),\left(\left(x_{3}, y_{3}\right),\left(x_{4}, y_{4}\right)\right) \in C_{x}$ and $\left(\left(x_{1}\right.\right.$, $\left.\left.y_{1}\right),\left(x_{2}, y_{2}\right)\right) \leq\left(\left(x_{3}, y_{3}\right),\left(x_{4}, y_{4}\right)\right)$ then:

$$
\begin{gathered}
\psi\left(\left(x_{1}, y_{1}\right),\left(x_{2}, y_{2}\right)\right)=\left(x_{1} \cap x_{2}, f\left(x_{1} \cap x_{2}\right)\right), \\
\psi\left(\left(x_{3}, y_{3}\right),\left(x_{4}, y_{4}\right)\right)=\left(x_{3} \cap x_{4}, f\left(x_{3} \cap x_{4}\right)\right) .
\end{gathered}
$$

$\left(\left(x_{1}, y_{1}\right),\left(x_{2}, y_{2}\right)\right) \leq\left(\left(x_{3}, y_{3}\right),\left(x_{4}, y_{4}\right)\right) \Rightarrow x_{1} \subseteq x_{3}, x_{2} \subseteq x_{4} \Rightarrow x_{1} \cap x_{2}$ $\subseteq x_{3} \cap x_{4} \Rightarrow\left(x_{1} \cap x_{2}, f\left(x_{1} \cap x_{2}\right)\right) \leq\left(x_{3} \cap x_{4}, f\left(x_{3} \cap x_{4}\right)\right) \Rightarrow$ $\psi\left(\left(x_{1}, y_{1}\right),\left(x_{2}, y_{2}\right)\right) \leq \psi\left(\left(x_{3}, y_{3}\right),\left(x_{4}, y_{4}\right)\right)$. So mapping $\psi$ is order-preserving.

Next, we proof the mapping $\psi$ is infimum-preserving.

Let $Y=\left\{\left(x_{t}, y_{t}\right) \mid T\right.$ is an index set, $\forall t \in T,\left(x_{t}, y_{t}\right)$ is a pair of concepts in direct product lattice $\left.L_{X} \cdot x_{t}=\left(p_{t}, q_{t}\right), y_{t}=\left(r_{t}, s_{t}\right)\right\}$, so $Y$ is a subset of $C_{X}$, then $\wedge Y=\left(\underset{t \in T}{\cap} x_{t}, f\left(\underset{t \in T}{\cap} x_{t}\right)\right)$.

$\left.\psi(\wedge Y)=\psi\left(\underset{t \in T}{\cap} x_{t}, f\left(\underset{t \in T}{\cap} x_{t}\right)\right)=\left(\underset{t \in T}{\cap} p_{t} \cap \underset{t \in T}{\cap} r_{t}\right), f\left(\underset{t \in T}{\cap} p_{t} \cap\left(\underset{t \in T}{\cap} r_{t}\right)\right)\right)$ $\psi(Y)=\psi\left(x_{t}, y_{t}\right)=\left\{\left(p_{t} \cap r_{t}, f\left(p_{t} \cap r_{t}\right)\right) \mid T\right.$ is an index set, $\forall t \in T$, $\left(x_{t}, y_{t}\right)$ is a pair of concepts in direct product lattice $\left.L_{X} \cdot\right\}$ $\wedge \psi(Y)=\left(\underset{t \in T}{\cap}\left(p_{t} \cap r_{t}\right), f\left(\underset{t \in T}{\cap}\left(p_{t} \cap r_{t}\right)\right)\right)=\left(\underset{t \in T}{\cap} p_{t} \cap\left(\underset{t \in T}{\cap} r_{t}\right), f\left(\underset{t \in T}{\cap} p_{t} \cap\right.\right.$ $\left.\left.\left(\underset{t \in T}{\cap} r_{t}\right)\right)\right)=\psi(\wedge Y)$.So, mapping $\varphi$ is infimum-preserving.

According to the above definition, theorem and proposition, may safely draw the following conclusion. The total lattice $L$ and the image on $L_{X}$ that are produced by mapping $\varphi$ are isomorphic. With this conclusion, to produce the total lattice $L$ by one sublattice of the direct product lattice $L_{X}$ whom satisfied some condition become possible.

Properties $1 \quad \forall c \in C, \varphi(c)=\min \left(\psi^{-1}(c)\right)$

Proof: Let $\left(\left(x_{1}, y_{1}\right),\left(x_{2}, y_{2}\right)\right)=\min \left(\psi^{-1}(c)\right), c=(p, q) \in C$.For any $\left(\left(\mathrm{s}_{1}, \mathrm{t}_{1}\right),\left(\mathrm{s}_{2}, \mathrm{t}_{2}\right)\right) \in\left(\psi^{-1}(c)\right)$ such that $\left(\left(x_{1}, y_{1}\right),\left(x_{2}, y_{2}\right)\right) \leq\left(\left(\mathrm{s}_{1}\right.\right.$, $\left.\left.\mathrm{t}_{1}\right),\left(\mathrm{s}_{2}, \mathrm{t}_{2}\right)\right)$.

(1)if $\left|\psi^{-1}(c)\right|=1 \quad$ then $\quad\left(\left(x_{1}, y_{1}\right),\left(x_{2}, y_{2}\right)\right)=\left(\left(\mathrm{s}_{1}, \mathrm{t}_{1}\right),\left(\mathrm{s}_{2}, \mathrm{t}_{2}\right)\right) \quad$.So properties 1 is tenable.

(2) if $\left|\psi^{-1}(c)\right| \neq 1$, then $\left(\left(x_{1}, y_{1}\right),\left(x_{2}, y_{2}\right)\right)<\left(\left(\mathrm{s}_{1}, \mathrm{t}_{1}\right),\left(\mathrm{s}_{2}, \mathrm{t}_{2}\right)\right)$.

Due to the" $\leq$ "relation in $L_{X} \Rightarrow y_{1} \supseteq \mathrm{t}_{1}, y_{2} \supseteq \mathrm{t}_{2}$. The mapping $\varphi$ is injective $\Rightarrow q \cap A_{1}=q \cap y_{1}, q \cap A_{2}=q \cap y_{2}$. So properties 1 is tenable.

Definition $8 \varphi^{\prime}$ :

$\forall c \in C, \varphi^{\prime}(c)=\left\{\left(c_{i}, c_{j}\right) \mid \operatorname{Extent}\left(c_{i}\right) \cap \operatorname{Extent}\left(c_{j}\right)=\operatorname{Extent}(c)\right\}$

Extent $(c)$ expresses the extent of concept $c$.The concepts of the total lattice $L$ can be computed easily by the image of the mapping $\varphi^{\prime}$.

Properties 2 For any concept $c=(x, y) \in C$, if $\varphi(c)=\left(\left(x_{1}\right.\right.$, $\left.\left.y_{1}\right),\left(x_{2}, y_{2}\right)\right)$ then $x=x_{1} \cap x_{2}$ and $y=y_{1} \cup y_{2}$.

Proof : Due to the define of $\varphi(c), y_{1}=y \cap A_{1}, y_{2}=y \cap A_{2}$ $\because \mathrm{A}_{1} \cap \mathrm{A}_{2}=\varnothing, \mathrm{A}_{1} \cup \mathrm{A}_{2}=\mathrm{A}, \therefore y=y_{1} \cup y_{2} \cdot \mathrm{g}\left(y_{1}\right)=\mathrm{x}_{1}, \mathrm{~g}\left(y_{2}\right)=\mathrm{x}_{2}$ then $\mathrm{g}(y)=\mathrm{g}\left(y_{1} \cup y_{2}\right)=\mathrm{g}\left(y_{1}\right) \cap \mathrm{g}\left(y_{2}\right)=\mathrm{x}_{1} \cap \mathrm{x}_{2}$.So properties 2 is tenable.

children $(c)$ expresses the set of the child concepts of concept $c$.It's elements are determined by the child concepts of $\varphi(c)$ whom is the image of concept $C$ in direct product lattice $L_{X}$.We get the images of $\varphi(c)$ 's child 
concepts by mapping $\psi$. The maximum of them form the set children $(c)$.

Definition 9 children $(c)=\max (\{\psi(\bar{c}) \mid \bar{c} \in$ children $(\varphi(c))\})$

If we want to build the total concept lattice with the original lattice and the additional lattice there are three questions need process.

(1)To find the update concepts from the direct product lattice that can produce new concepts.

(2)To compute the extent and the intent of these concepts.

(3)To define the father-son relation between these concepts.

(1)(2)(3) are the main steps of this algorithm and the above properties form the theory foundation. This algorithm can produce the total concept lattice only through the direct product operation on the original lattice and the additional lattice.

Input: direct product lattice $L_{X}$, the result of direct product operation on The original lattice $L_{1}$ and the additional lattice $L_{2}$.

Output: $L$, the total concept lattice $L$

Procedure Constrct-lattice (Input ; $L_{X}$,Output : $L$ )

Begin

$$
L \leftarrow \varnothing \text {; }
$$

For each $\left(c_{i}, c_{j}\right)$ in $C_{1} \times C_{2}$ do

$$
\begin{aligned}
& E \leftarrow \operatorname{Extent}\left(c_{i}\right) \cap \operatorname{Extent}\left(c_{j}\right) \\
& \text { Judgevalue } \leftarrow \psi\left(\operatorname{children}\left(c_{i}, c_{j}\right)\right)
\end{aligned}
$$

// If this concept is an update concept

If Findnew(E,Judgevalue) then

$/ /$ produce a new concept $C$. Its extent is $E$ and intent is the union of $C_{i}$ 's intent and $C_{j}$ 's intent.

$$
c \leftarrow\left(E \text {,Intent }\left(c_{i}\right) \cup \operatorname{Intent}\left(c_{j}\right)\right)
$$

For each $\bar{c}$ in $\max ($ Judgevalue) do

$\operatorname{Link}(c, \bar{c}) / / \bar{c}$ is $c$ 's child concept. Link $\bar{c}$ to $c$

$L \leftarrow L \cup\{c\}$ //Add the new concept $c$ to the $L$

End

//To judge whether $\left(c_{i}, c_{j}\right)$ is updated concept. If the return value is true then $\left(c_{i}, c_{j}\right)$ is updated concept.

Produce Findnew(E, Judgevalue)

Begin

$$
\begin{aligned}
& \text { flag } \leftarrow \text { false // signal variable } \\
& \text { For each } \bar{c} \text { in Judgevalue do } \\
& \text { If Extent }(\bar{c}) \neq E \text { then } \\
& \text { flag } \leftarrow \text { true }
\end{aligned}
$$

Else begin

$$
\text { flag } \leftarrow \text { false }
$$$$
\text { //if Extent }(\bar{c})=E \text { then break the circulation and }
$$
return the flag 's value. return flag end

return flag
End

// the algorithm get the child concepts of $\left(c_{i}, c_{j}\right)$

Procedure children $\left(c_{i}, c_{j}\right)$

Begin

child $\leftarrow \varnothing$

For each $\left(c_{m}, c_{n}\right)$ in $C_{1} \times C_{2}$ do

If $\left(c_{m}, c_{n}\right)<\left(c_{i}, c_{j}\right)$ and has no $\left(c_{k}, c_{r}\right)$ such that

$\left(c_{m}, c_{n}\right)<\left(c_{k}, c_{r}\right)<\left(c_{i}, c_{j}\right)$ then

$/ /\left(c_{m}, c_{n}\right)$ is child concept of $\left(c_{i}, c_{j}\right)$

child $\leftarrow$ child $\cup\left\{\left(c_{m}, c_{n}\right)\right\}$

children $\leftarrow$ child

End

\section{EXAMPLE}

Here, we use a specific example to demonstrate the algorithm. The original formal context $\left(O, A_{1}, I_{1}\right)$.The additional formal context $\left(O, A_{2}, I_{2}\right)$. Let $O=\{1,2\}, A_{1}=\{a, b, c\}$, $A_{2}=\{d, e\}$.These formal contexts and their concept lattices are shown as Figure 1.The total formal context $(O, A, I)$ is shown as Figure 2.

The lattice generated by direct product operation on two of the original formal context concept lattice is shown on the left in Figure 3.

Using the above algorithm on this lattice, firstly find the update concepts, secondly according to the properties 2 calculated the concept $C$ of the combined total lattice. Finally, according to the definition 9 found child nodes of concept $c$ and connecting them. It constructed a combined total concept lattice (such as the right side of Figure 3 shows).
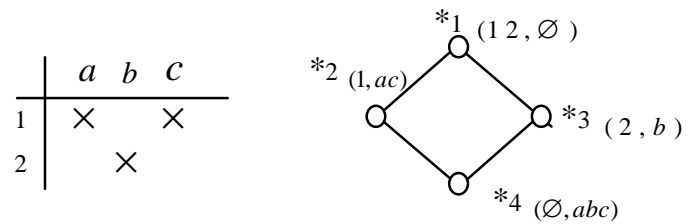

(a) The original formal context and its concept lattice

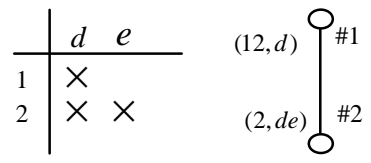

(b) The additonal formal context and its concept lattice

Figure 1.The original formal context, the additional formal context and their corresponding concept lattice 


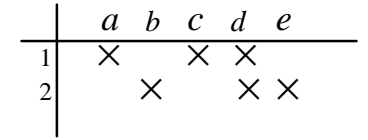

Figure 2.The incorporative formal context

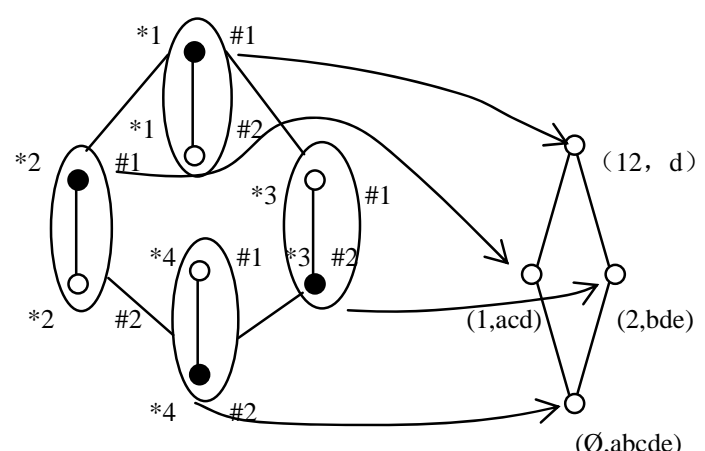

Figure 3 The direct product lattice $L_{x}$ 、 mapping $\psi$ and the incorporative concept lattice $L$

In order to verify the correctness of the algorithm, we compared this algorithm and the algorithm that produces concept lattice by formal context with experiments. The latter's input is the total formal context and the output is the total concept lattice. The program use VB6.0 as programming language. Experiment data use random data.

The number of objects is set to 30 . The number of attributes is changing, from the beginning of 20, incrementing by 20 until 200. Each object has the same number of attributes. Context concentration is $40 \%$. The experimental results prove the validity of the algorithm.

Algorithm features: Some of the existing incremental construction algorithms of concept lattice, their core idea is with a concept lattice as the basis, then every increase a attribute to traverse the concept lattice one time[12,13].

According to the additional attributes to determine concept category, and carries on the corresponding operation to form the new concept lattice until you add all attributes to generate the final result.

This algorithm provides a new method with the direct product for constructing concept lattice. Firstly, we produce the direct product lattice. Using the original concept lattice and direct product lattice isomorphic relation, can easily determine the father-son relationship of concepts in the direct product concept lattice. Concepts are only divided into two categories: update and non-update. The operation is simple.

The method only needs to traverse the direct product lattice one time. In the traverse, it adds all additional attributes and generates the concept lattice from the bottom to the top. It does not traverse and operate the concept lattice when there is a new attribute is added like the existing incremental algorithms. So, it is more efficient than the incremental algorithm.

\section{CONCLUSION}

Concept lattice generation problem is the key steps of knowledge extracting from database. To overcome the shortcomings of the present incremental building algorithms of concept lattice, we propose a new method based on direct product. It is applicable nicely when a large number attributes were added to database or more than two database were merged. The experiment proved that the algorithm is correct, but the application scope is limited. The next improvements include reducing the limitations of the algorithm, and to expand the scope of application of the algorithm, to reduce the search range of the update concept, as well as to improve the association rules extraction algorithm in data mining.

\section{REFERENCES}

[1] Wille $\mathrm{R}$. Restructuring lattice theory: an approach based on hierarchies of concepts. Rival I(ed).Ordered Sets. Dordrecht-Boston: Reidel Publishing Company, 1982. 445 470.

[2] R Godin, G Mineau, R Missaoui, et al, “Applying concept formation methods to software reuse”,International Journal of Knowledge Engineering and Software Engineering, 1995, 5(1): 119 142.

[3] G W Mineau, R Godin, "Automatic structuring of knowledge bases by conceptual clustering”, IEEE Transaction on Knowledge and Data Engineering, 1995, 7(5): 824 828.

[4] R Cole, P Eklund, "Scalability in formal concept analysis", Computational Intelligence, 1999,15 (1): 11 27.

[5] C Carpineto, G Romano, "A lattice conceptual clustering system and its application to browsing retrieval”,Machine Learing, 1996, 24(2): 95 122. minutes

[6] R Godin, R Missaoui, “An incremental concept formation approach for learning from databases”,Theoretical Computer Science, 1994, 133(5): 387 419.

[7] J P Bordat, "Calcul pratique du trellis de galois d'une correspondence”, Mathematiques et Sciences, 1986, 96: 31 47.

[8] Lhouari Nourine, Olivier Raynaud, "A fast algorithm for building lattices”,Information Processing Letters, 1999, 71(5-6): 199 204.

[9] R Godin, "Incremental concept formation algorithms based on Galois (concept) lattices”,Computational Intelligence, 1995, 11(2): 246 267.

[10] Z.P. Xie, L.Z. Tian,“A Fast Incremental Algorithm for Building Concept Lattice”, Chinese Journal of Computers, 2002, 25(5): 490 496.

[11] Ganter B, Wille R. Formal concept analysis: mathematical foundations. Berlin: Springer, 1999.

[12] S.Q. Jian, X.G. Hu, M.H. Jiang, "Incremental Algorithms of Extended Concept Lattice ”, Computer Engineering and Applications 2001, 36(15): 132 134.

[13] X.J. Shen, D.J. Han, Z.T. Liu, Jun Ma, “Improvement on Constructing Algorithm of Concept Lattices”, Computer Engineering and Applications, 2004, 40(24): 100 104. 